\title{
Where Physics Meets Biology
}

\author{
MARIO NICODEMI \\ mario.nicodemi@unina.it \\ University of Naples Federico II
}

\begin{abstract}
The frontier between life and physical sciences currently includes the strategic research field where the wealth of data produced by new quantitative technologies in molecular biology naturally meets the advanced analysis and modelling tools of theoretical physics. For its profound scientific implications and huge potential impacts in biomedicine, such an area of investigation is attracting substantial interest. Therefore, in the following contribution, I briefly review some developments in the research field under scrutiny.
\end{abstract}

\section{Introduction}

Molecular Biology has made impressive scientific advances in the last decades, with a sequence of paradigm-shifting breakthroughs occurring every few years, as perhaps happened in Physics at the beginning of the last century with the development within just 30 years of quantum, relativistic and statistical mechanics. However, the interest of theoretical physicists in molecular biology began right after those years. It is little known, for instance, that Richard Feynman, who would win the Nobel Prize in Physics in 1965 for his studies on elementary particles (for the theory of quantum electro-dynamics), devoted himself in the late 1950s and early 1960s to work on genetics with Robert S. Edgar in Max Delbruck's lab at Caltech. In the mid-1960s, Feynman acknowledged the significant biology progresses also in his famous textbook, Lectures on Physics, where he wrote (in Feynman et al., 2005 [1964]):

[...] certainly no subject or field is making more progress on so many fronts at the present moment, than biology, and if we were to name the most powerful assumption [...] to understand life, it is that all things are made of atoms, and that everything that living things do can be understood in terms of [...] atoms.

Feynman's (in Feynman et al., 2005 [1964]) statement encapsulates the very ambitious project to explain biological phenomena from the fundamental principles of physics, as done for other many-body systems of nature, from superconductivity to soft-matter physics because, ultimately, biological systems are made of atoms and, therefore, must obey the laws of physics.

The ideal project summarised by Feynman (in Feynman et al., 2005 [1964]) is being materialised in recent years because of the substantial technological advancements made in molecular biology. These new technologies produce highly replicable, quantitative experimental data on biological systems at the single molecule level. The wealth and complexity of those data naturally require advanced analysis tools and models from hard 
sciences to be interpreted and understood. For such a reason, the science at the frontier between molecular biology and theoretical physics has become a strategic research field.

\section{Where Theoretical Physics meets Molecular Biology}

The completion in 2001 of the Human Genome Project, for example, has provided the details of the sequence of the human genome and, in particular, of our genes. The fundamental question, however, on how the system works remains open. Why is a gene active in certain tissues and not in others? Why an oncogene, remained silent for years, suddenly becomes active in a cell leading to cancer? Quantitative experimental data of the post-genomic era are opening the way to answering those questions by combining biology and the methods of physics because, after all, the human genome is only one of the complex systems of physics, as highlighted by Feynman (in Feynman et al., 2005 [1964]). The answers to those questions will explain the functioning of life itself and will usher in new approaches to personalised medicine for the treatment of diseases such as cancer or congenital disorders.

In recent years, it has been discovered, for example, that the human genome has a complex spatial organisation within the cell nucleus that serves vital functional purposes (Dekker, 2016; Dixon, 2016; Sigal, 2018; Finn, 2019; Kempfer, 2019). In order to regulate their activity, genes must establish physical contact with remote regulatory regions along the DNA, millions of bases away, that control transcription. The result is a complex architecture in which our 20,000 genes are brought into contact with their corresponding regulators (it is estimated that each gene has on average four remote DNA regulatory sequences) in an impressive example of selforganisation. The complex three-dimensional structure of our DNA defines the activity and, therefore, the fate of a cell by establishing the genes to be activated and those to be silenced. Alterations of the genome spatial organisation can modify gene expression and lead to disease (Spielmann, 2018).

The study of the spatial architecture of chromosomes is a perfect ground where theoretical physics has fruitfully met biology because chromosomes are polymers, and physics naturally intervenes in the description and understanding of polymer behaviours (de Gennes, 1979). My research group, for example, has worked to develop new methods to experimentally measure the structure of chromosomes with high precision (Beagrie, 2017; Fiorillo, 2021) and to understand, via Statistical Mechanics, the physical mechanisms that shape their threedimensional conformation and bring together genes and their regulators (Barbieri, 2012; Barbieri, 2017; Bianco, 2018; Conte, 2020).

\section{The mechanisms that regulate our genome}

The abovementioned discoveries on the genome's architecture have radically changed the way we look at our DNA. They have shown that, in order to understand how the system works, we must not only decipher its linear sequence of bases (i.e., the ATCG letters of its alphabet), but we must also understand its complex three-dimensional structure, that is, how the DNA folds on itself in the space of the nucleus.

Genes are essential because they encode the production of proteins, the building blocks of cells to function. However, it is less known that they comprise only $2 \%$ of the human genome 
length. The role of the remaining $98 \%$ has been mysterious for years. It was named 'junk DNA' according to the idea that it is a useless relic left by millions of years of evolution. However, it is now established that, far from being junk, it contains, for instance, the remote sequences that regulate genes and, therefore, the key to gene functioning (Andersson, 2014). We have also discovered that our DNA is folded in the cell nucleus in a hierarchical structure made of domains-within-domains (Fraser, 2015), like Chinese boxes, where the complicated interactions between genes and their regulators take place. Additionally, those interactions have been shown to typically involve the simultaneous colocalisation of different chromosomal regions adding another level of complexity to the system (Beagrie, 2017).

We are also starting to understand the physical mechanisms that define the threedimensional structure of the genome, that is to say, how and why regulatory contacts are formed. We have discovered that they are based, for example, on thermodynamic phase transitions involving the DNA polymer and a number of molecular factors with which it interacts. Coil-to-globule transition mechanisms serve, for instance, to establish contacts between remote sequences (Barbieri, 2012; Bianco, 2018), while phase-separation mechanisms are used to isolate distinct regions that should not interact (Barbieri, 2017; Conte, 2020). In this way, we begin to understand why and how genetic mutations that do not affect gene integrity can lead to disease: they can alter the three-dimensional structure of the DNA by modifying the network of regulatory contacts between genes and regulators, hence producing incorrect activations or repressions (Bianco, 2018; Dellino, 2019).

\section{Discussion}

While the discoveries at the frontier between molecular biology and physics are unveiling the very functioning of life, the scientific and technological advances made in recent years have the potential to revolutionise medical applications far beyond basic science. Combined with the clinical use of the new powerful DNA sequencing methods, they can be used, for instance, to predict the medical implications of mutations in single patients and even in single cells. We are beginning to understand the complex origin of genetic diseases such as congenital disorders (Bianco 2018; Kragesteen 2018) or cancer (Dellino, 2019). These discoveries are paving the way for the development of new diagnostic and treatment methods. These advances are part of the current broader scientific revolution taking place at the frontier between life and hard sciences, which will provide a fundamental understanding of the mechanisms of life and will revolutionise biomedical and clinical applications for the generations to come.

All significant technological developments, ranging from nuclear energy to artificial intelligence, raise high hopes for a better life for humankind as well as concerns and ethical issues. Human knowledge and scientific progress must not be stopped for no reason. Nevertheless, practical applications of those discoveries ought to be carefully evaluated and planned as they may challenge fundamental human values and the very essence of human existence. For example, as we delve into the mechanisms of genetics, we can interfere at an unprecedented scale with the course of life of single individuals, populations and, more broadly, with human evolution. That can erode one of the very founding concepts of modern nations and civilizations whereby equal rights are granted to all. As novel clinical methods require advanced and expensive technologies, they might become available to only the more 
affluent nations or wealthier individuals, independent of universal rights or individual merits. To avoid turning hope into despair and unrest, I think all measures must be implemented to account for ethical issues and deliver progress to humankind.

\section{Keywords}

technology management; ethical organisations; physics; biology

\section{References}

Andersson, R., Gebhard, C., Miguel-Escalada, I., Hoof, I., Bornholdt, J., Boyd, M., Chen, Y., Zhao, X., Schmidl, C., Suzuki, T., Ntini, E., Arner, E., Valen, E., Li, K., Schwarzfischer, L., Glatz, D., Raithel, J., Lilje, B., Rapin, N., Bagger, F.O., Jørgensen, M., Andersen, P.R., Bertin, N., Rackham, O., Burroughs, A.M., Baillie, J.K., Ishizu, Y., Shimizu, Y., Furuhata, E., Maeda, S., Negishi, Y., Mungall, C.J., Meehan, T.F., Lassmann, T., Itoh, M., Kawaji, H., Kondo, N., Kawai, J., Lennartsson, A., Daub, C.O., Heutink, P., Hume, D.A., Jensen, T.H., Suzuki, H., Hayashizaki, Y., Müller, F., The FANTOM Consortium, Forrest, A.R.R., Carninci, P., Rehli, M., and Sandelin, A. (2014), “An Atlas of Active Enhancers across Human Cell Types and Tissues", Nature, 507: 455-461. DOI: 10.1038/nature12787.

Barbieri, M., Chotalia, M., Fraser, J., Lavitas, L.M., Dostie, J., Pombo, A., and Nicodemi, M. (2012), "Complexity of Chromatin Folding Is Captured by the Strings and Binders Switch Model", Proceedings of the National Academy of Sciences Oct. 2012, 109 (40): 1617316178. DOI: 10.1073/pnas.1204799109.

Barbieri, M., Xie, S.Q., Triglia, E.T., Chiariello, A.M., Bianco, S., de Santiago, I., Branco, M.R., Rueda, D., Nicodemi, M., and Pombo, A. (2017), “Active and Poised Promoter States Drive Folding of the Extended HoxB Locus in Mouse Embryonic Stem Cells", Nature Structural \& Molecular Biology, 24 (6): 515-524. DOI: 10.1038/nsmb.3402.

Beagrie, R.A., Scialdone, A., Schueler, M., Kraemer, D.C.A., Chotalia, M., Xie, S.Q., Barbieri, M., de Santiago, I., Lavitas, L.M., Branco, M.R., Fraser, J., Dostie, J., Game, L., Dillon, N., Edwards, P.A.W., Nicodemi, M., and Pombo, A. (2017), “Complex Multi-Enhancer Contacts Captured by Genome Architecture Mapping (GAM), a Novel Ligation-Free Approach", Nature, 543: 519-524. DOI: 10.1038/nature21411.

Bianco, S., Lupiáñez, D.G., Chiariello, A.M., Annunziatella, C., Kraft, K., Schöpflin, R., Wittler, L., Andrey, G., Vingron, M., Pombo, A., Mundlos, S., and Nicodemi, M. (2018), “Polymer Physics Predicts the Effects of Structural Variants on Chromatin Architecture", Nature Genetics, 50: 662-667. DOI: 10.1038/s41588-018-0098-8.

Conte, M., Fiorillo, L., Bianco, S., Chiariello, A.M., Esposito, A., and Nicodemi, M. (2020), "Polymer Physics Indicates Chromatin Folding Variability across Single-Cells Results from State Degeneracy in Phase-Separation", Nature Communications, 11 (1): 3289-3295. DOI: 10.1038/s41467-020-17141-4.

de Gennes, P.G. (1979), Scaling Concepts in Polymer Physics, Ithaca/London: Cornell University Press.

Dekker, J., and Mirny, L. (2016), “The 3D Genome as Moderator of Chromosomal Communication", Cell, 164 (6): 1110-1121. DOI: 10.1016/j.cell.2016.02.007. 
Dellino, G.I., Palluzzi, F., Chiariello, A.M., Piccioni, R., Bianco, S., Furia, L., De Conti, G., Bouwman, B.A.M., Melloni, G., Guido, D., Giacò, L., Luzi, L., Cittaro, D., Faretta, M., Nicodemi, M., Crosetto, N., and Pelicci, P.G. (2019), "Release of Paused RNA Polymerase II at Specific Loci Favors DNA Double-Strand-Break Formation and Promotes Cancer Translocations", Nature Genetics, 51: 1011-1017. DOI: 10.1038/s41588-019-0421-z.

Dixon, J.R., Gorkin, D.U., and Ren, B. (2016), “Chromatin Domains: The Unit of Chromosome Organization”, Molecular Cell, 62 (5): 668-680. DOI: 10.1016/j.molcel.2016.05.018.

Feynman, R.P., Leighton, R.B., and Sands, M. (1964, revised edn. 2005), The Feynman Lectures on Physics, Reading, MA: Addison-Wesley.

Finn, E.H., and Misteli, T. (2019), "Molecular Basis and Biological Function of Variability in Spatial Genome Organization”, Science, 365 (6457). DOI: 10.1126/science.aaw9498.

Fiorillo, L., Musella, F., Conte, M., Kempfer, R., Chiariello, A.M., Bianco, S., Kukalev, A., Irastorza-Azcarate, I., Esposito, A., Abraham, A., Prisco, A., Pombo, A., and Nicodemi, M. (2021), "Comparison of the Hi-C, GAM and SPRITE Methods Using Polymer Models of Chromatin", Nature Methods, 18: 482-487. DOI: 10.1038/s41592-021-01135-1.

Fraser, J., Ferrai, C., Chiariello, A.M., Schueler, M., Rito, T., Laudanno, G., Barbieri, M., Moore, B.L., Kraemer, D.C.A., Aitken, S., Xie, S.Q., Morris, K.J., Itoh, M., Kawaji, H., Jaeger, I., Hayashizaki, Y., Carninci, P., Forrest, A.R.R., The FANTOM Consortium, Semple, C.A., Dostie, J., Pombo, A., and Nicodemi, M. (2015), "Hierarchical Folding and Reorganization of Chromosomes Are Linked to Transcriptional Changes in Cellular Differentiation", Molecular Systems Biology, 11 (12): 852-858. DOI: 10.15252/msb.20156492.

Kempfer, R., and Pombo, A. (2019), "Methods for Mapping 3D Chromosome Architecture", Nature Reviews Genetics, 21 (4): 207-226. DOI: 10.1038/s41576-019-0195-2.

Kragesteen, B.K., Spielmann, M., Paliou, C., Heinrich, V., Schöpflin, R., Esposito, A., Annunziatella, C., Bianco, S., Chiariello, A.M., Jerkovic, I., Harabula, I., Guckelberger, P., Pechstein, M., Wittler, L., Chan, W.L., Franke, M., Lupiáñez, D.G., Kraft, K., Timmermann, B., Vingron, M., Visel, A., Nicodemi, M., Mundlos, S., and Andrey, G. (2018), "Dynamic 3D Chromatin Architecture Contributes to Enhancer Specificity and Limb Morphogenesis", Nature Genetics, 50: 1463-1468. DOI: 10.1038/s41588-018-0221-x.

Sigal, Y.M., Zhou, R., and Zhuang, X. (2018), “Visualizing and Discovering Cellular Structures with Super-Resolution Microscopy", Science, 361 (6405): 880-887. DOI: 10.1126/science.aau1044.

Spielmann, M., Lupianez, D.G., and Mundlos, S. (2018), "Structural Variation in the 3D Genome", Nature Reviews Genetics, 19: 453-467. DOI: 10.1038/s41576-018-0007-0. 